\title{
Alterações morfológicas da glândula supra-renal na síndrome da imunodeficiência adquirida
}

\author{
Adrenal gland morphological alterations in the acquired \\ immunodefficiency syndrome \\ Francisco Moretti Duch, Cibele Alvarenga Répele, Fernanda Spadaro, \\ Marlene Antônia dos Reis, Denise Bertulucci Rocha Rodrigues, \\ Mara Lúcia da Fonseca Ferraz e Vicente de Paula Antunes Teixeira
}

\begin{abstract}
Resumo Na síndrome da imunodeficiência adquirida (AIDS) pode-se verificar o acometimento da supra-renal por efeito citopático direto pelo HIV, por infecções oportunistas ou neoplasias. Estes achados poderiam variar de acordo com a procedência do paciente, devido às doenças peculiares à região. Neste trabalho avaliou-se o comprometimento da supra-renal em quatorze pacientes que morreram de AIDS no Hospital Escola, em Uberaba. Treze eram do sexo masculino e treze brancos. A idade foi de 29,9 \pm 7,8 anos e o índice de massa corporal foi de $19 \pm 4,1 \mathrm{~kg} / \mathrm{m}^{2}$. Os fragmentos de supra-renal obtidos nas necropsias foram analisados em microscópio de luz. Encontramos inflamação em 100\% dos casos, identificando-se o agente etiológico em oito $(58,1 \%)$ casos. O Citomegalovírus foi identificado em sete casos, o Cryptococcus sp e o Herpes simplex em dois e o Histoplasma sp em um caso, estes achados são semelhantes aos da literatura. Em um caso, encontramos calcificação do parênquima e em outro, flebite da veia central. Em alguns casos que apresentavam lesão não foi possível identificar o agente etiológico, talvez em decorrência do efeito citopático direto pelo HIV ou devido a toxicidade das drogas utilizadas no tratamento da AIDS e das infecções oportunistas.
\end{abstract}

Palavras-chaves: AIDS. Infecções oportunistas. Necropsia. Supra-renal.

\begin{abstract}
In the acquired immunodeficiency syndrome (AIDS), the adrenal glands are subject to opportunistic infections, neoplasm or direct cytopathic effect by HIV. It is know that the incidence and type of adrenal involvement vary according to the patient's place of origin. In this paper we evaluate adrenal involvement in fourteen patients that died from AIDS in the University Hospital of Uberaba, Brazil. The group studied was comprised of thirteen males and thirteen whites. The age was $29.9 \pm 7.8$ years, and the body mass index was $19.0 \pm 4.1 \mathrm{~kg} / \mathrm{m}^{2}$. Adrenal specimens obtained from autopsies were analyzed by light microscopy. Inflammation was found in $100 \%$ of the cases and the etiologic agent(s) was (were) identified in eight (58.1\%) patients. Citomegalovirus was identified in seven cases, Cryptococcus $\mathrm{sp}$ and Herpes simplex in two and Histoplasma $\mathrm{sp}$ in one case, these pathologic findings were similar to literature. We also found parenchymal calcification and adrenal central vein phlebitis in one case each. Injury was found in some cases without indentified infections agent. This fact could be due to the direct cytopathic effect by HIV, or due to toxicity of drug therapy used during treatment of AIDS and opportunistic infections.
\end{abstract}

Key-words: Adrenal gland. AIDS. Autopsy. Opportunistic infections.

Disciplinas de Patologia Geral da Faculdade de Medicina do Triângulo Mineiro e da Universidade de Uberaba, Uberaba, Minas Gerais. Apoio financeiro: Fundação de Ensino e Pesquisa de Uberaba (FUNEPU), Fundação de Amparo a Pesquisa do Estado de Minas Gerais (FAPEMIG) e Conselho Nacional de Desenvolvimento Científico e Tecnológico (CNPq).

Endereço para correspondência: Prof. Vicente de Paula Antunes Teixeira. Disciplina de Patologia Geral/FMTM. R. Frei Paulino 30, 38025-180 Uberaba, MG.

E-mail: vicpat@mednet.com.br

Recebido para publicação em 21/08/97. 
As glândulas supra-renais são órgãos endócrinos complexos, pesando no adulto de 4 a $5 \mathrm{~g}$. Nos indivíduos falecidos após longos períodos de enfermidade elas podem estar alteradas, presumivelmente como resultado do estímulo do ACTH induzido pelo estresse 3 . A homeostase do organismo está relacionada, em grande parte, à função da supra-renal, particularmente devido aos hormônios e outros peptídeos produzidos nesta glândula7.

Nos EUA, mais de $80 \%$ dos casos de insuficiência da supra-renal são devidos à destruição auto-imunitária da glândula. É comum a presença de anticorpos dirigidos contra o córtex supra-renal e há evidências de anormalidade da imunidade celular13. Com exceção da tuberculose e da paracoccidioidomicose, são raras as inflamações que causam destruição da glândula a ponto de provocar hipocorticalismo. De fato, considera-se suficiente a conservação de um décimo da glândula para suprir as exigências do organismo. Antigamente a tuberculose era a causa mais comum de insuficiência crônica da supra-renal, representando agora apenas 10 a $20 \%$ dos casos. A histoplasmose pode também provocar destruição acentuada das suprarenais 2 . Já publicou-se também o acometimento desta glândula em várias doenças, tais como, na raiva, na sífilis, na criptococose, na toxoplasmose e na doença de Chagas 1412.

Atualmente estudam-se as alterações na supra-renal em decorrência da síndrome da imunodeficiência adquirida (AIDS), dentre elas, as manifestações endócrinas causadas pelo HIV e o efeito citopático direto pelo vírus, principalmente na medular devido ao tropismo do HIV pelo tecido neural. Destacam-se ainda as infecções oportunistas, principalmente pelo Citomegalovírus (CMV) e Mycobacterium avium. O mesmo autor chama a atenção também para - acometimento da própria glândula por neoplasias, p. ex., o sarcoma de Kaposi, além das alterações causadas por efeitos colaterais das drogas utilizadas no tratamento destes indivíduos ${ }^{9}$. Tem sido descrito ainda necrose e trombose de pequenos vasos tanto na cortical como na medular8. Nestes estudos, as manifestações podem variar de acordo com a procedência dos pacientes. Provavelmente isto se deva as doenças endêmicas ou não, peculiares àquelas regiões onde foram realizados os trabalhos.

O objetivo do presente trabalho foi avaliar através de métodos morfológicos o comprometimento da supra-renal dos pacientes com AIDS necropsiados em Uberaba, Minas Gerais.

\section{MATERIAL E MÉTODOS}

Em um estudo retrospectivo, foram revistos quatorze protocolos de um total de 221 necropsias, realizadas no período de 1990 a 1997, no Hospital Escola da Faculdade de Medicina do Triângulo Mineiro, em Uberaba (MG). Copilamos dos registros: a cor, a idade, o sexo e calculamos o índice de massa corporal (IMC), dado por peso corporal em quilogramas dividido pela altura expressa em metros ao quadrado.

Durante a necropsia foram coletados pelo menos dois fragmentos, sendo um de cada supra-renal. Estes foram processados rotineiramente em parafina, e as lâminas foram analisadas ao microscópio de luz. Além da coloração pela Hematoxilina-eosina, nos casos indicados realizamos as colorações histoquímicas suplementares necessárias (Picrosírius, Impregnações argênticas, Mucicarmin de Mayer).

\section{RESULTADOS}

A idade variou de 20 a 44 anos, com média de 29,9 $\pm 7,1$ anos. Dos casos analisados treze eram do sexo masculino, treze brancos e apenas um não-branco. O IMC variou de $13,8 \mathrm{~kg} / \mathrm{m}^{2}$ a $28,6 \mathrm{~kg} / \mathrm{m}^{2}$, com média de $19,0 \pm 4,1 \mathrm{~kg} / \mathrm{m}^{2}$.

Quanto aos processos patológicos observamos a inflamação, constituída por infiltrado predominantemente de mononucleares e escassos polimorfonucleares, em todos os casos. A hipotrofia foi encontrada em treze $(92,8 \%)$ casos e pela necrose em nove $(64,3 \%)$ casos. Em três casos encontramos fibrose da cortical e medular, sendo que em um deles estava associada a calcificação do parênquima. Em um caso encontramos flebite da veia central da supra-renal (Tabela 1).

O sítio mais comum de inflamação foi a medula, seguido do córtex e depois a cápsula (Tabela 2). Foi possível identificar o agente etiológico em oito $(58,1 \%)$ deles. Encontramos o CMV em sete casos, o Cryptococcus sp e o Herpes simplex em dois e o Histoplasma sp em um. Em dois casos o CMV e o Cryptococcus sp estavam presentes concomitantemente, e em dois outros casos, o CMV e o Herpes simplex (Tabela 3). 
Tabela 1 - Processos patológicos encontrados nas supra-renais de pacientes com AIDS necropsiados.

\begin{tabular}{lcc}
\hline Processos patológicos & \multicolumn{2}{c}{ Pacientes } \\
\cline { 2 - 3 } & $\mathrm{n}^{0}$ & $\%$ \\
\hline Inflamação & 14 & 100,0 \\
Hipotrofia & 13 & 92,8 \\
Necrose & 9 & 64,3 \\
Congestão & 9 & 64,3 \\
Hemorragia & 4 & 28,6 \\
\hline Observação: A soma das porcentagens ultrapassa 100\% pois mais de um processo patológico foi encontrado no \\
mesmo corte da supra-renal. \\
Além dos processos patológicos descritos na tabela encontramos: \\
$\quad$ Em dois casos, edema da cápsula fibrosa \\
$\quad$ Em três casos, fibrose da córtex e medula, sendo que em um caso estava associada extensa área de \\
$\quad$ calcificação. \\
$\quad$ Em um caso, flebite da veia central da supra-renal \\
$\quad$ Em dois casos, trombose na veia central e/ou tributária
\end{tabular}

A hipotrofia global ocorreu em treze $(92,8 \%)$ casos, sendo que em doze deles o córtex foi a camada mais acometida (Tabela 2). A hemorragia observada em quatro $(28,6 \%)$ casos, apresentouse com intensidade discreta ou moderada, sendo que em um caso estava presente tanto na cápsula como na medula e no córtex. Em dois casos a hemorragia foi observada somente na medula e em um caso, somente na cápsula (Tabelas 1 e 2).

Tabela 2 - Processos patológicos mais comuns e os segmentos topográficos mais acometidos nas supra-renais de pacientes com AIDS necropsiados.

\begin{tabular}{|c|c|c|c|c|c|c|c|c|}
\hline & \multicolumn{2}{|c|}{ Inflamação } & \multicolumn{2}{|c|}{ Hipotrofia } & \multicolumn{2}{|c|}{ Hemorragia } & \multicolumn{2}{|c|}{ Congestão } \\
\hline & $\mathrm{n}^{\circ}$ & $\%$ & $\mathrm{n}^{0}$ & $\%$ & $\mathrm{n}^{\circ}$ & $\%$ & $\mathrm{n}^{\circ}$ & $\%$ \\
\hline Córtex & 8 & 57,2 & 12 & 92,3 & 1 & 25,0 & 5 & 55,5 \\
\hline Medula & 11 & 78,6 & 2 & 15,4 & 3 & 75,0 & 8 & 88,9 \\
\hline Cápsula & 4 & 28,6 & - & - & 2 & 50,0 & 2 & 22,2 \\
\hline
\end{tabular}

Observação: A soma das porcentagens ultrapassa $100 \%$ pois mais de um processo patológico foi encontrado no mesmo corte da suprarenal.

Tabela 3 - Relação da inflamação com o agente infeccioso nas supra-renais de pacientes com AIDS necropsiados.

\begin{tabular}{lrr}
\hline \multicolumn{1}{c}{ Agentes } & \multicolumn{2}{c}{ Pacientes } \\
\cline { 2 - 3 } & $\mathrm{n}^{\mathbf{0}}$ & $\%$ \\
\hline "Agente não classificado" & 6 & 42,8 \\
CMV & 7 & 50,0 \\
Cryptococcus sp & 2 & 14,3 \\
Herpes simplex & 2 & 14,3 \\
Histoplasma sp & 1 & 7,1
\end{tabular}

Observação: A soma das porcentagens ultrapassa $100 \%$ pois mais de um agente infeccioso foi encontrado no mesmo corte da supra-renal.

\section{DISCUSSÃO}

Nos quatorze pacientes necropsiados estudados encontramos inflamação em 100\% dos casos, identificando-se o agente etiológico em oito $(58,1 \%)$ deles. O CMV foi identificado em sete casos, o que representa $50 \%$ da casuística. Este resultado é semelhante aos descritos por Marks9, e muito próximo do encontrado no estudo feito por Gasglow e cols5. Estes últimos mostraram $51 \%$ de infecção pelo CMV em 41 casos de necropsias dos pacientes HIV positivos estudados, sendo a medula mais freqüentemente acometida pela inflamação que 
o córtex. Estes dados também estão de acordo com o encontrado no nosso estudo, onde observamos $78,6 \%$ de acometimento da medula e $57,1 \%$ de acometimento do córtex. Em apenas um caso verificamos flebite da veia central da supra-renal, aparentemente em continuidade com a medulite. Este achado embora ainda não descrito em caso de AIDS, é semelhante ao encontrado em chagásicos crônicos ${ }^{12}$.

Marks ${ }^{9}$, mostrou em uma série de necropsias que a supra-renal é a glândula endócrina mais freqüentemente acometida na AIDS, e que o CMV é o agente infeccioso mais freqüente, causando desde supra-renalite focal até necrose extensa. Outro achado importante mencionado pela autora é de inflamação da supra-renal sem um agente infeccioso detectado. Este fato tem sido interpretado como evidência de inflamação nesta topografia causada diretamente pelo HIV, pelo conhecido tropismo do vírus pelo tecido nervoso.

As manifestações endócrinas na AIDS estão associadas a vários mecanismos, incluindo efeito direto do retrovírus, infecções oportunistas, neoplasias, alteração na nutrição e os efeitos das drogas usadas no tratamento da doença. Neste último aspecto, sabe-se que o cetoconazol, agente antifúngico, inibe a esteroidogênese. $A$ rifampicina aumenta o risco de insuficiência adrenal por aumentar a atividade do citocromo P-450 e alterar o metabolismo do cortisol. A suramina, agente antiviral, interfere na síntese de glicocorticóides e leva a processo degenerativo no córtex da supra-renal. O uso da vidarabina exacerba a hiponatremia do paciente, assim como o ganciclovir inibe a esteroidogênese e a pentamidina leva a hipoglicemia9.

Desses dados da literatura, podemos inferir que o efeito das drogas se associa ao próprio quadro anátomo-clínico da doença, podendo levar a alterações na supra-renal por ação direta. Entre estas alterações, a calcificação por nós encontrada talvez se encaixe dentro deste mecanismo patogenético. Outra possível causa de calcificacão na glândula é a pneumocistose disseminada10, evento não encontrado no nosso caso.

Contribui também para a insuficiência suprarenal, os processos anatomopatológicos causados por agentes oportunistas, sendo que independentemente da região geográfica, o CMV é o agente mais encontrado. A suprarenalite por CMV é caracterizada pela inclusão intranuclear e intracitoplasmática patognomônicas desta infecção. Ela afeta preferencialmente a camada medular, levando a necrose coagulativa. O grau de necrose da supra-renal correlacionase com o grau de envolvimento pelo CMV5.

Vários investigadores relatam uma relação in vitro entre o Herpes simplex (HSV), o CMV e a replicação do HIV. As primeiras proteínas reguladoras do HSV e do CMV parecem induzir a ativação de longa repetição terminal do HIV e o aumento da transcrição, resultando no aumento da produção de RNA viral. Especula-se que a resposta imune contra o HSV ou o CMV induziria maior produção de citocinas que potencializariam um aumento na replicação do HIV11.

Em infecções generalizadas não há tendência ao acometimento da supra-renal. Entretanto, uma vez estabelecida, a lesão nesta glândula se torna maior do que as lesões extra suprarenais, especialmente quando se trata da infecção por HSV, CMV e Herpes zoster 4 .

Em nosso estudo encontramos $42,8 \%$ dos casos de inflamação, sem a evidência do agente infeccioso. Este processo inflamatório pode ser devido ao efeito citopático direto do HIV sobre a glândula, ou talvez seria por ação de outro agente infeccioso não detectável pelas colorações utilizadas no presente estudo. Outra hipótese para explicar as alterações degenerativoinflamatórias das supra-renais, seria devido ao emprego de drogas para o tratamento dos agentes infecciosos ou mesmo da própria AIDS9.

Além disso, Groll e cols6, estudando a hipófise e a supra-renal de 130 necropsias descreveram anormalidades em $76 \%$ destas. As lesões predominantes foram inflamação e infiltração por sarcoma de Kaposi ou linfoma não-Hodgkin. $\mathrm{O}$ achado mais comum foi suprarenalite necrosante por CMV, em $52 \%$ dos casos. Em um terço destes, levou a intensa destruição envolvendo mais de $50 \%$ do parênquima. A destruição quase total das supra-renais, em alguns casos, sugerem que a supra-renalite por CMV pode apresentar curso destrutivo progressivo.

$O$ estado terminal dos pacientes com AIDS freqüentemente é marcado por hipotensão, hiponatremia e hipovolemia, sugerindo o comprometimento da supra-renal. Este comprometimento pode ser caracterizado por alterações necrótico-degenerativas, acompanhadas por inflamação na glândula. Tais processos devem-se em parte aos agentes infecciosos 
freqüentemente encontrados, ao próprio HIV ou mesmo devido às drogas usadas no tratamento destes pacientes.

\section{AGRADECIMENTOS}

Os autores agradecem a Vandair Gonçalves, Maria Prado de Morais e Aloísio Costa, pelos serviços técnicos prestados.

\section{REFERÊNCIAS BIBLIOGRÁFICAS}

1. Almeida HO, Teixeira VPA, Oliveira G, Brandão MC, Gobbi H. Medulite supra-renálica em casos de raiva humana. Memórias do Instituto Oswaldo Cruz 81:439-442, 1986.

2. Bisi H. Cortical da supra-renal. In: Brasileiro Filho G, Pittella JEH, Pereira FEL, Bambirra EA, Barbosa AJA (eds) Bogliolo Patologia. 5 ${ }^{\mathrm{a}}$ edição. Guanabara Koogan, Rio de Janeiro p. 930-942, 1994.

3. Cotran RS, Kurman V, Robins S. Córtex supra-renal. In: Cotran RS, Kurman V, Robins S (eds) Robins Patologia Estrutural e Funcional. 5 a edição. Guanabara Koogan, Rio de Janeiro p. 1036-1048, 1996.

4. Frenkel JK. Infections involving the adrenal córtex. In: Moon HD (ed) The adrenal cortex. Harper and Brothers, 1961.

5. Glasgow BJ, Kenneth DS, Anders K, Layfield LJ. Adrenal pathology in the acquired immune deficiency syndrome. American Journal of Clinical Pathology 84: 594-597, 1985.

6. Groll A, Schneider M, Althoff PH, Falckenbach A,
Helm EB, Keul HG, Schleiblinger S, Hübner K. Morphologie und klinische bedeutung pathologischer veränderungen an nebennieren und hypophyse bei AIDS. Deutsche Medizinische Wochenschrift 115:483-488, 1990.

7. Junqueira LC, Carneiro J. Adrenais. In: Junqueira LC, Carneiro J (eds) Histologia Básica, 6 edição. Guanabara Koogan, Rio de Janeiro p. 399-425, 1991.

8. Laulund S, Vesfeldt J, Klinken L. PhatoAnatomical studies in patients dying of AIDS. Acta Pathologic Microbiologic Imunologic Scandinavia 94:201-221, 1986.

9. Marks JB. Endocrine manifestations of human immunodeficiency virus (HIV) infection. The American Journal of Medical Sciences 302:110-117, 1991.

10. Radin DR, Baker EL, Klatt EC, Balthazar EJ, Jeffrey RB Jr, Megibow AJ, Ralls PW. Visceral and nodal calcification in patients with AIDS-related Pneumocystis carinii infection. American Journal of Roentgenology 154: 27-31, 1990.

11. Stein DS. Herpes virus infeccion, HIV, and disease progression. AIDS Clinical Care 7:11-17,1995.

12. Teixeira VPA, Gobbi H, Almeida HO. Supra-renalite em chagásicos crônicos. Revista da Sociedade Brasileira de Medicina Tropical 18:155-159, 1985.

13. Tirrell JB, Hipofunção córtico supra-renal. In: Wingaarden JB, Smith LH, Bennet JC (eds) Cecil Tratado de Medicina Interna, 19a edição. Guanabara Koogan, Rio de Janeiro, vol. 2, p. 